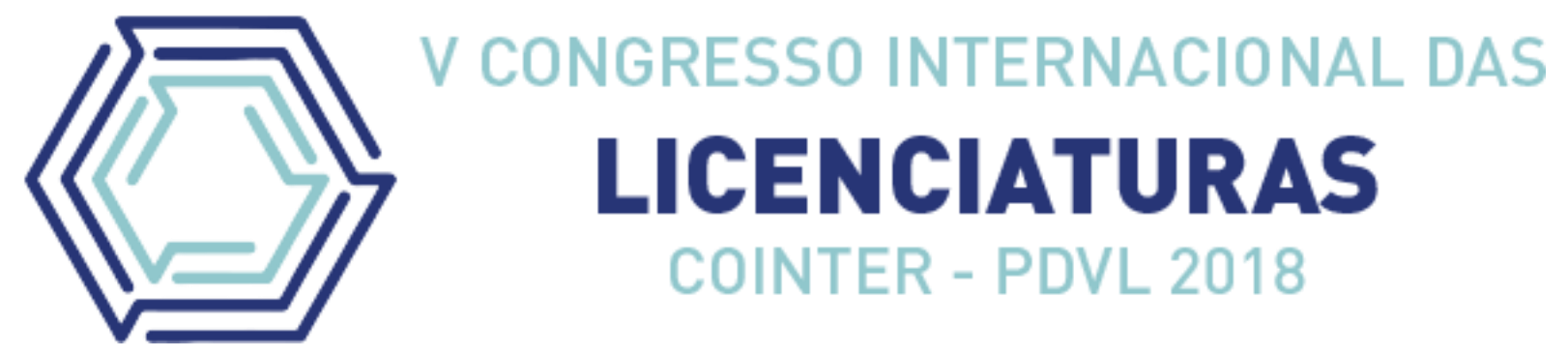

\title{
PROCESSO DE RECICLAGEM PARA PRODUÇÃO DE SACOLAS PLÁSTICAS E OS IMPACTOS GERADOS AO MEIO AMBIENTE
}

\section{RECYCLING PROCESS FOR THE PRODUCTION OF PLASTIC BAGS AND THE IMPACTS GENERATED TO THE ENVIRONMENT}

\author{
Apresentação: Comunicação Oral \\ SANTOS, Ronaldo José ${ }^{1}$; JESUS, Maioneide Madalena ${ }^{2}$; HOMERO, Jail ${ }^{3}$ LOPES Antônio \\ Marcelo ${ }^{4}$ SÁ, Ézio Raul Alves de ${ }^{5}$
}

DOI: https://doi.org/10.31692/2358-9728.VCOINTERPDVL.2018.00132

\section{Resumo}

Atualmente o excesso de resíduos poliméricos no meio ambiente vem crescendo em larga escala, devido ao seu descarte incorreto. Uma das alternativas para diminuir esse problema ambiental é a reciclagem destes polímeros. Através da conscientização da população, com campanhas educativas e investimentos em coleta seletiva e em aterros sanitários bem equipados, além de incentivos fiscais para as empresas que realizam o processo de reciclagem. Com isto, o trabalho visa analisar o processo de reciclagem de plásticos em uma fábrica de sacolas localizada complexo industrial no bairro Pantanal na cidade de Picos - PI. Através de um questionário proposto à gerente de produção da fábrica buscando conhecer todo o processo de fabricação, desde a chegada da matéria-prima, da separação dos termoplásticos, até chegar no produto final que é as sacolas e também o destino final do produto. Na qual, utilizam-se da reciclagem de matéria-prima proveniente da coleta tanto da macrorregião, como oriundas de outros estados como: Ceará, Pernambuco, para obtenção de um novo produto, essas sacolas biodegradáveis que são distribuídas pela macrorregião de Picos. Mas a maior parte da produção vai para o Maranhão e o Pará. Com isso, foi possível analisar a importância da fábrica para a região de Picos, como a retirada do lixo das ruas e a criação de dezenas de empregos direto e indiretamente movimentando a economia da cidade, e também é crucial ao meio ambiente pois o tempo de degradação desses polímeros é muito demorado. Causando a poluição do solo e podendo contaminar o lençol freático da região causando impactos alarmantes.

Palavras-Chave: Resíduos, Polímeros, Reciclagem, Fabricação de Sacolas, impactos ambientais.

\footnotetext{
${ }^{1}$ Química, IFPI campus picos, ronaldo.j.s2007@gmail.com

2 Química, IFPI campus picos, maioneidejesus19@gmail.com

${ }^{3}$ Química, IFPI campus picos, jailhomero@outlook.com

${ }^{4}$ Química, IFPI campus picos, antonio_marcelo05@gmail.com

${ }^{5}$ Mestrado, IFPI campus picos, ezio.sa@ifpi.edu.br
} 


\begin{abstract}
Currently the excess of polymeric residues in the environment has been growing on a large scale due to its incorrect disposal. One of the alternatives to reduce this environmental problem is the recycling of these polymers. Through the awareness of the population, educational campaigns and investments in selective collection and well-equipped landfills, as well as tax incentives for companies that carry out the recycling process. With this, the work aims to analyze the plastic recycling process in an industrial complex located in the Pantanal neighborhood in the city of Picos - PI. Through a questionnaire proposed to the production manager of the plant seeking to know the entire manufacturing process, from the arrival of the raw material, to the separation of the thermoplastics, until arriving at the final product that is the bags and also the final destination of the product. In that, they use the recycling of raw material from the collection of the macro-region, as well as from other states such as: Ceará, Pernambuco, to obtain a new product, these biodegradable bags that are distributed in the macro-region of Picos. But most of the production goes to Maranhão and Pará. With this, it was possible to analyze the importance of the factory for the Picos region, such as the removal of garbage from the streets and the creation of dozens of jobs directly and indirectly moving the economy of the city, and is also crucial to the environment because the degradation time of these polymers is very time consuming. Causing soil pollution and can contaminate the water table of the region causing alarming impacts.
\end{abstract}

Keywords: Waste, Polymers, Recycling, Bag Making, Environmental Impacts.

\title{
Introdução
}

As questões ambientais assumiram nos últimos anos lugar central no discurso e na agenda de trabalho de diferentes segmentos da sociedade. Isso devido ao impacto que os resíduos causam ao meio ambiente e demonstrando como é preocupante a importância dos resíduos sólidos para reciclagem, sendo que estes materiais demoram muito tempo para degradarem no ambiente (COHEN, 2001 in apud DIAS \& TEODOSIO, 2006).

O Brasil produz diariamente cerca de 149 mil toneladas de resíduos sólidos, mas apenas 13,4 mil, ou 9\%, são recicladas, segundo o Informe Analítico da Situação da Gestão Municipal de Resíduos Sólidos no Brasil, do Ministério das Cidades (IDEC, 2006). O restante, 135,6 mil toneladas, é destinado a aterros sanitários (32\%), aterros clandestinos (59\%) ou lançados diretamente nas ruas e terrenos baldios, causando problemas ao meio ambiente e gerando sérios riscos à saúde pública (DIAS \& TEODOSIO, 2006).

Os plásticos constituem uma das classes de materiais com menor índice de reciclagem, muitas das vezes, relacionado com o valor pago pelo volume coletado, outras vezes pela dificuldade de se coletar grandes quantidades. Os metais (latinhas, entre outros) coletados, 
possuem um valor comercial melhor, e mais fácil de realizar o manuseio, e ser enviado para os depósitos de reciclagem, por isso se torna um dos "preferidos" para coleta, e reciclagem (CZAPSKI, 2003 in apud DIAS \& TEODOSIO, 2006).

Outro problema relacionado aos resíduos plásticos é o tempo de degradação, estes resíduos levam muito tempo para sofrerem degradação espontânea, e quando queimados, produzem gases tóxicos. Por isso o excesso deste material no solo, prejudica muito o ambiente (MANO, BONELLI, 1994; ZANIN, MANCINI, 2004). A reciclagem surge como uma das vias para reduzir este tipo de material aterrado no solo, causando sua poluição, prejudicando, inclusive, o tempo de vida útil dos aterros sanitários, e consequentemente ocupando mais espaço físico urbano (DIAS \& TEODÓSIO, 2006).

Diante do exposto acima o trabalho busca compreender todo o processo envolvido no processo de fabricação, apresentando a relevância da reciclagem de sacolas, reutilização dos plásticos e a importância da fábrica de sacolas para melhoria da qualidade do meio ambiente. Como também os benefícios que a fábrica propicia aos moradores da região de Picos- PI.

\section{Fundamentação Teórica}

Nas últimas décadas, os plásticos revolucionaram as nossas vidas (THOMPSON et al., 2009). Na primeira página do livro "Plastics", YARSLEY e COUZENS (1945) apud THOMPSON et al., (2009) afirmam que "as possíveis aplicações (dos plásticos) são praticamente inexauríveis”. Isso é uma realidade hoje em dia, pois os plásticos estão presentes em praticamente todos os setores da sociedade, incluindo roupas, artigos esportivos, edificações, automóveis, aviões, aplicações médicas, entre outros. A diversidade e a versatilidade dos polímeros facilitam a produção de uma enorme variedade de produtos que proporcionam avanços tecnológicos, economia de energia e diversos outros benefícios para a sociedade (ANDRADY e NEAL, 2009).

O primeiro plástico sintético foi desenvolvido no início do século $\mathrm{XX}$, e registrou um desenvolvimento acelerado a partir de 1920. Este material, relativamente novo se comparado a outros como o vidro e o papel, passou a estar presente em grande parte dos nossos utensílios. O plástico vem das resinas derivadas do petróleo e pertence ao grupo dos polímeros (moléculas muito grandes, com características especiais e variadas). Os materiais plásticos são polímeros 
formados a partir de combinação de moléculas, pela indústria petroquímica, que originam diferentes tipos de plásticos e possuem alto peso molecular (AGUILAR-VIRGEN et al., 2010). Conforme a configuração específica dos polímeros, eles se dividem em termoplásticos, termorrígidos (termofixos) e elastômeros (borrachas) (GORNI, 2003).

O plástico, inventado pelo inglês Alexander Parkes em 1862 em Trigueiro (2003), possui propriedades que são fundamentais atualmente. São leves, resistentes, maleáveis e impermeáveis. Surgem às sacolas plásticas, que foram introduzidas nos anos 70, e se tornaram populares pela distribuição gratuita a cada compra em supermercados e lojas (FABRO, 2007).

Uma das implicações que existe no aumento da utilização do plástico consiste na durabilidade do material, o que ocasiona um alto tempo de decomposição pelo meio ambiente, ou seja, a maior parte dos plásticos usados não são biodegradáveis. Uma das alternativas a esse problema é a reutilização de materiais plásticos (AGUILAR-VIRGEN et al., 2010).

As sacolas plásticas demoram cerca de 200 anos para se decompor quando estão soterradas no lixo, e quando ficam expostas a radiação solar, geralmente se decompõem em um ano (ZIEGLER, 2010). Assim, a demora na deterioração deste material não é o maior de todos os problemas ambientais causados por ele. O grande problema centra-se no processo de fabricação das sacolas de polietileno, uma vez que este material, derivado do petróleo, é altamente poluente, nocivo à fauna e a flora.

Atualmente, muito se discute sobre o uso correto das sacolas plásticas. Em alguns estados brasileiros, já é Lei o uso das sacolas “oxi-biodegradáveis”. Muitos supermercados aderiram ao uso de sacolas de plástico oxi-degradável, adotando-as como forma alternativa ao uso de sacolas plásticas descartáveis. Estas sacolas recebem um aditivo no momento da produção que acelera sua degradação. Assim, a sacola deve se decompor em no máximo 18 meses (ZIEGLER, 2010; PORTAL EDUCAÇÃO, 2012). Entretanto, o problema não é somente o tempo que este material demora em se decompor, mas sim, a maneira como ele é descartado, pois as substâncias resultantes de sua decomposição também são altamente poluentes.

As sacolas plásticas também contribuem para o aquecimento global, porque os processos de refino do petróleo e fabricação das sacolas consomem energia, água, liberam 
efluentes e emitem gases poluentes. Cem milhões de sacolas plásticas precisam de 1,5 milhões de litros de petróleo para serem produzidas e causam a emissão de 4,2 mil toneladas de CO2 (PLANTIER, 2013).

A legislação ambiental brasileira sobre o gerenciamento de resíduos sólidos consiste, inicialmente, na classificação dos resíduos considerados perigosos, com base na origem, características e demais aspectos com impactos potenciais. Na última década, a legislação brasileira tem considerado, de forma inovadora em relação aos demais países, a responsabilidade do gerador ao longo da cadeia produtiva (BRASIL, 1998, CONAMA, 2000 e Rodrigues, 2002), estabelecendo-se regras para as operações de tratamento, estocagem e disposição destes resíduos para que práticas inadequadas de gerenciamento de resíduos possam ser identificadas e eliminadas.

Nesta parte do artigo, o autor deve fazer uma exposição e uma discussão das teorias que foram utilizadas para entender e esclarecer o problema, apresentando-as e relacionando-as com a dúvida investigada. A fundamentação apresentada servirá de base para as análises dos dados, no momento da apresentação e discussão dos resultados.

\section{Metodologia}

O presente artigo analisou através de uma pesquisa quali-quantitativa, com objetivos de natureza e descritiva a importância da realização de uma pesquisa local em uma fábrica de sacolas, na qual faz a reciclagem de plásticos na cidade de Picos - PI. A coleta de dados foi feita durante o mês de julho de 2018, onde foram realizadas 3 visitas na fábrica, a qual tem atuação na mesma cidade há cerca de 7 anos.

No primeiro momento da visita, no dia 14/07 não houve muito sucesso, devido à falta de disponibilidade de tempo por parte dos funcionários, marcando assim para outro momento. Na segunda visita, que aconteceu no dia 17/07 conheceu - se as dependências da fábrica, bem como os funcionários da mesma e responsável em geral. Na terceira visita realizada no dia 19/07, foi aplicado um questionário com questões abertas para um dos funcionários da empresa, visando um conhecimento mais aprofundado sobre a mesma e os processos ali desenvolvidos. 
As visitas tiveram como finalidade avaliar os processos de obtenção da matéria-prima, estrutura da fábrica e o processamento da mesma para obtenção do produto final explicados pelos funcionários da respectiva entidade. Avaliou-se também o valor agregado ao produto e o impacto social, para que assim compreenda a importância da reciclagem de resíduos plásticos para o meio ambiente.

\section{Resultados e Discussão}

Após a realização das visitas a uma fábrica na cidade de Picos pode-se perceber que a mesma tem grande importância para a população dessa microrregião como também para o meio ambiente, pois é uma empresa sustentável, onde a matéria prima utilizada é material reciclado e produz plásticos biodegradáveis, os quais possuem um processo de decomposição mais rápido.

E como afirma Alencar (2005), a reciclagem dos plásticos é muito importante, pois pode reduzir a quantidade de lixo nos aterros sanitários e serve como matéria-prima para ser reaproveitada e fazer novos produtos, trazendo muitos benefícios para a população, contribuindo para a limpeza da cidade, e também pode gerar mais empregos, diminui a poluição e o consumo de energia. E com isso melhora o ambiente em que vivemos, podendo ver o lixo, com novas utilidades, não causando uma ameaça.

Segundo Oliveira (2012), a reciclagem de plásticos também é apontada por diversos autores como um processo eficaz para a redução e reaproveitamento de resíduos plásticos, com menor uso de energia e geração de emissões quando comparada à fabricação da resina virgem. Existe, entretanto, um enorme potencial de aumento das quantidades de resina que podem ser recicladas, se forem tomadas medidas para que este objetivo possa ser atingido.

Para a pesquisa aplicou-se um questionário sobre a produção e o destino dos resíduos provenientes da fabricação das sacolas. Onde foi investigado como a coleta era realizada, se a empresa terceirizava esse serviço de coleta e a frequência de como era feita.

A responsável pelo processo de produção afirmou que a própria empresa tem uma equipe destinada apenas para coleta e que esse processo é realizado todos os dias, geralmente sendo transportado por caminhões que percorrem toda a macrorregião de Picos e estados vizinhos. 
Após coletados, os resíduos são transportados para uma unidade de triagem, equipada com lugares para catação, para que seja feita uma separação mais criteriosa dos materiais visando à comercialização deles. É importante que a população seja devidamente orientada para que somente separe como lixo seco os materiais que possam ser comercializados, evitando-se despesas adicionais com o transporte e manuseio de rejeitos, que certamente são produzidos durante o processo de seleção por tipo de material e no enfardamento (MONTEIRO et al., 2001).

Posteriormente questionou-se sobre o tipo de material reciclado e como era feita a separação de outros tipos de plásticos coletados. Pode-se constatar que boa parte do material recolhido pela coleta tem outros destinos, apenas os termoplásticos são mais largamente utilizados, por que são materiais que podem ser reprocessados várias vezes pelo mesmo ou por outro processo de transformação, quando submetidos ao aquecimento a temperaturas adequadas. Segundo a entrevistada esse processo de separação é feito manualmente e só posteriormente a esse é que a resina virgem é lavada e colocada no moinho onde sofre trituração e transformação em pequenos grãos de polietileno.

Um ponto mais relevante foi quando questionou se as sacolas produzidas eram biodegradáveis e o tempo que as mesmas permanecem no meio ambiente. Na qual obteve-se como resultado de que as sacolas são biodegradáveis. O processo de biodegradação baseia-se em três estágios: o primeiro refere-se ao início do processo de fabricação, onde se introduz o aditivo específico à resina plástica, estabelecendo-se assim a vida útil do produto.

O segundo estágio relaciona-se ao final da vida útil, onde o produto já começa a degradar-se na presença de oxigênio por um processo de oxidação acelerado pela luz e calor, fazendo com que a cadeia molecular do polímero plástico seja quebrada em cadeias moleculares menores. Por fim, a biodegradação é completada pelos microrganismos sem deixar resíduos nocivos, como metano, pois o processo todo ocorre na presença de oxigênio (RES BRASIL, 2013).

O tempo esperado para decomposição dos plásticos biodegradáveis pode variar de dias até anos, dependendo dos requisitos do produto, ou seja, irá depender das formulações de aditivos e seus diferentes níveis de inclusão no produto final (POLIFILME, 2013).

Em seguida perguntou-se quais os polímeros usados na fabricação das sacolas e a entrevistada não soube responder ao certo, o que nos levou a uma pesquisa mais aprofundada na própria fábrica, descobrindo assim que o principal polímero é polietileno de baixa densidade. 
Mais esse polímero utilizado para fabricação de sacolas plásticas no Brasil nem sempre é o mesmo dependendo do fabricante. O polímero utilizado pode variar entre o Polietileno de Baixa densidade (PEBD ou LDPE) e o Polietileno de Alta Densidade (PEAD ou HDPE), podendo também ocorrer à mistura de ambos (COUTINHO et al., 2003).

E a última indagação, teve como propósito analisar através do entrevistado, quais as vantagens de reciclar esses materiais para o meio ambiente. A funcionária brevemente afirmou que é algo necessário para todos, pelo fato de retirar produtos inutilizáveis do meio. E que deve ter uma maior conscientização da população a respeito da importância da reciclagem não só dos plásticos mais como todo material que é descartado nos lixões.

A reciclagem, evita a poluição do ambiente, isto é da água, ar e solo, provocada pelo lixo, e isto faz aumentar a vida útil dos aterros sanitários, diminuindo, a quantidade de resíduos sólidos, e também diminui a exploração dos recursos naturais, os quais não são renováveis como o petróleo. Com isso, gera oportunidades de empregos para a população não qualificada e estimula a concorrência, pois os produtos feitos com materiais reciclados são comercializados em paralelos àqueles feitos com matérias-primas nunca utilizadas. Além disso, diminui os gastos com a limpeza urbana, e também com a poluição ambiental (SCARLATO e PONTIN, 1992).

\section{Conclusões}

Ao analisar o meio em que vivemos, é perceptível o acúmulo de resíduos sólidos ou poliméricos, em aterros sanitários, encostas de rios e/ou ao ar livre. Acarretando ao meio ambiente e à vida humana diversas conturbações. Portanto, com a pesquisa realizada na fábrica de sacolas, notou-se a importância de algumas atividades, como a reciclagem feita pela mesma. Que pode proporcionar emprego, além de garantir ao mercado consumidor produtos biodegradáveis e com menos tempo de decomposição, no presente caso, as sacolas.

A fabricação de sacolas é realizada durante várias etapas e processos que vai desde a sua coleta, separação, lavagem, secagem, trituração, aquecimento até chegar ao produto final de interesse. A fabricação constitui-se de um processo no qual transforma matéria-prima coletada por diversas regiões, em produto final destinado a toda macrorregião de Picos e estados circunvizinhos. Além de conhecer cada processo envolvido, desde a reciclagem até a obtenção das sacolas, foi possível compreender a importância da fábrica na cidade e que medidas podem 
ser tomadas, para diminuir o número de lixo plástico descartado de maneira inadequada no meio ambiente.

Para minimizar problemas ambientais referentes ao acúmulo de lixo em áreas desapropriadas, algumas iniciativas podem ser tomadas como, a implantação de coletas seletivas, a realização de campanhas informativas de conscientização junto à população, convencendo-a da importância da reciclagem, orientando-a para a separação correta do lixo, de acordo com o tipo de material, a prática de campanhas educativas, atividades de reciclagem, entre outras são medidas positivas, que juntamente com a ação governamental e social podem trazer resultados significativos para o meio ao qual estamos inseridos. Contudo, a conscientização da sociedade é primordial para que questões ambientais sejam mais facilmente resolvidas.

\section{Referências}

AGUILAR-VIRGEN, Q.; VEJA, C.A.; TABOADA-GONZÁlEZ, P.; AGUILAR, X.M., Potencial de recuperación de residuos sólidos domésticos dispuestos em um rellenosanitario. Revista de Ingeniería, Universidade de losAndes, Bogotá, Colombia, p.1627, 2010.

ALENCAR, M.M. M. Reciclagem de Lixo numa escola pública do Município de Salvador. Revista Virtual, v. 1, n.2, p. 96-113, 2005.

ANDRADY, A. L., NEAL, M. A., "Applications and Societal Benefits of Plastics", Philosophical Transactions of the Royal Society B, v. 364, pp. 1977-1984, 2009.

CONAMA, 2000. Resolução $\mathrm{n}^{\circ}$ 265, de 27 de janeiro de 2000. Disponível em: <http://www.lei.adv.br/265-00.html> Acessado em agosto de 2018.

COUtinho, F. M. B.; MEllo, I. L.; MARIA, L. C. de S. Polietileno: Ciência e Tecnologia, Vol 13, nº1. Rio de Janeiro, 13 p., 2003. 
FABRO, A. T.; LINDEMANN, C.; VIEIRA, S.C. Utilização de sacolas plásticas em supermercados.Revista Ciências do Ambiente On-Line. v.3, n.1, p. 15-23, 2007.

DIAS ,G. S.L.F.and TEODOSIO, A. dos S.S. Estrutura da cadeia reversa: "caminhos" e "descaminhos" da embalagem PET.Prod. [online]., vol.16, n.3 pp. 429-441. 2006. Acesso em 20 de julho de 2018.

GORNI, A. A. Introdução aos Plásticos[2003]. Revista plástico industrial. Disponível em: <http://www.gorni.eng.br/intropol.html $>$ Acesso em: 20 de julho de 2018.

MANO, E. B.; BONELli, C. M. C. A Reciclagem de plásticos pós-consumidos.Rev. Química. Industrial. Rio de Janeiro, n. 698, p. 18-22, 1994.

MONTEIRO, J. H. P. et al. Manual de Gerenciamento Integrado de Resíduos Sólidos. Rio de Janeiro: Instituto Brasileiro de Administração Municipal, 2001.

OLIVEIRA, M. C. B. R., Gestão de Resíduos Plásticos Pós-Consumo: Perspectivas para a Reciclagem no Brasil, 2012. Dissertação (mestrado).Rio de Janeiro:UFRJ/COPPE. p.7686,2012 .

POLIFILME embalagens. Degradação de sacolas plásticas convencionais e oxibiodegradáveis [2013]. Revista do Centro de Ciências Naturais e Exatas - UFSM. Disponível em: < https: //periodicos.ufsm.br/cienciaenatura/article/viewFile/24176/pdf > Acesso em 27 de Julho de 2018.

PlantiER, R. D. Como é Produzido o Plástico Biodegradável[2013]. Disponível em: http://meioambiente.culturamix.com/gestaoambiental/como-e-produzido-o-plasticobiodegradavel Acesso em: 25 de julho de 2018.

PORTAL EDUCAÇÃO. Sacolas Plástica x Meio Ambiente: Como re(agir)?. Portal Educação: $\quad$ [s.l.], 2012. Disponível em: <http://www.portaleducacao.com.br/biologia/artigos/14622/sacolas-plastica-X-meioambiente-como-reagir\#ixzz2IFMeCQf4>. Acesso em: 20 de julho de 2018. 
RES BRASIL. Degradação de sacolas plásticas convencionais e oxibiodegradáveis [2013]. Revista do Centro de Ciências Naturais e Exatas - UFSM. Disponível em: < https:// periodicos.ufsm.br/cienciaenatura/article/viewFile/24176/pdf > . Acesso 27 de Julho de 2018.

RODRIGUES, G.A. Ação civil pública e termo de ajustamento de conduta: teoria e prática. Rio de Janeiro: Forense, 2002.

SCARlato, F. C.; PONTIN, J. A. Do Nicho ao Lixo. Ambiente, Sociedade e Educação. São Paulo: Atual, 1992.

THOMPSON, R. C, MOORE, C. J, SAAL, F.S, SWAN, S. H,"Plastics, the environment and human health: current consensus and future trends", PhilosophicalTransactionsofthe Royal Society B, n. 364, pp. 2153-2166, 2009.

TRIGUEIRO, A. A farra dos sacos plásticos. 2003. Disponível em: $<$ http://www.jardimdeflores.com.br/ECOLOGIA/A33sacolas_plasticas.html >. Acesso em: 27 jul. 2018.

ZIEGLER, M. F. Por que a sacola de plástico é prejudicial ao meio ambiente: Entenda o problema das sacolas plásticas e quais as alternativas ambientalmente corretas para transportar suas compras [2010]. iG: São Paulo. Disponível em: $<$ http://ultimosegundo.ig.com.br/ciencia/meioambiente/por-que-a-sacola-de-plastico-eprejudicial-ao-meio-ambiente/n1237730908104.html>. Acesso em: 20 de julho de 2018. 